\title{
Semiconductor Quantum Dots are Efficient and Recyclable Photocatalysts for Aqueous PET-RAFT Polymerization
}

\author{
Kevin P. McClelland, ${ }^{\ddagger}$ Tristan D. Clemons, ${ }^{\ddagger}$ Samuel I. Stupp, ${ }^{\dagger £ § \| \#}$ and Emily A. Weiss ${ }^{* \ddagger \uparrow}$ \\ ‡Department of Chemistry, and ॠCenter for Light Energy-Activated Redox Processes (LEAP), Northwestern \\ University 2145 Sheridan Road, Evanston, IL 60208, United States \\ ${ }^{\dagger}$ Departments of Materials and Science \& Engineering, ${ }^{\S}$ Medicine, "Biomedical Engineering, 2220 Campus Drive, \\ Evanston, IL 60208, United States; \#Simpson Querrey Institute, Northwestern University, 303 East Superior Street, \\ Chicago, IL 60611, United States. \\ *corresponding author. Email: e-weiss@northwestern.edu
}

\section{Supporting Information}

\section{Table of Contents:}

\section{Experimental Procedures and Calculations}

Materials

Experimental techniques

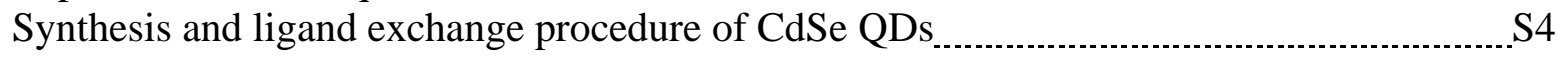

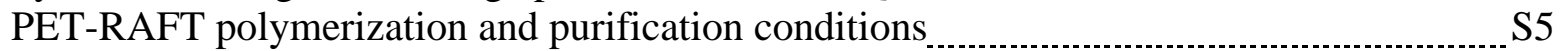

\section{Supporting Figures}

Figure S1. PL of oleate- and MPA-capped QDs _._. S5

Figure S2 ${ }^{1} \mathrm{H}$ NMR of DMA polymerization kinetics with QDs

Figure S3. Absorbance spectrum of Eosin Y before and after illumination _........................S7

Figure S4. ${ }^{1} \mathrm{H}$ NMR of DMA polymerization Without QDs …………………......... S7

Figure S5. ${ }^{1} \mathrm{H}$ NMR of DMA polymerization kinetics with Eosin Y

Figure S6. ${ }^{1} \mathrm{H}$ NMR of interrupted DMA polymerization kinetics with QDs ........................S8

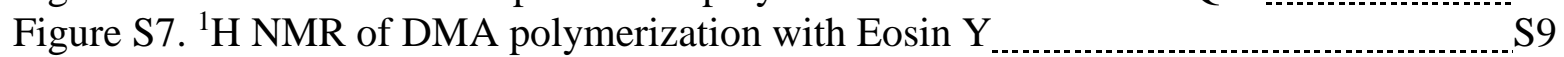

Figure S8. ${ }^{1} \mathrm{H}$ NMR of DEA polymerization with QDs _........................................ 9

Figure S9. ${ }^{1} \mathrm{H}$ NMR of AMP polymerization with QDs $\ldots$

Figure S10. ${ }^{1} \mathrm{H}$ NMR of HEA polymerization with QDs $\ldots \ldots \ldots$

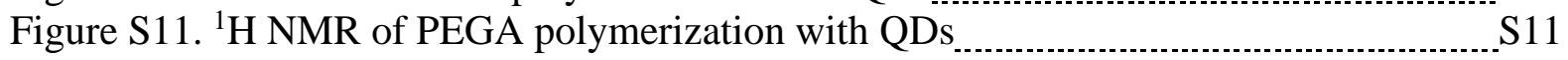

Figure S12. GPC of polymerization with QDs for all monomers _.................................S11

Figure S13. ${ }^{1} \mathrm{H}$ NMR of pDMA purification with $3 \mathrm{kDa}$ protein concentrators _................ S12

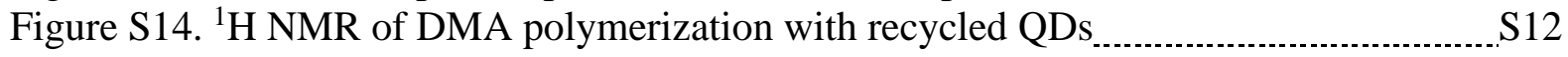

Figure S15. GPC of DMA polymerization with recycled QDs _._. S13 


\section{EXPERIMENTAL METHODS}

Materials. Oleic acid (OA, 90\%), 1-octadecene (ODE, 90\%), Cadmium oxide (CdO, 99.99\%), selenium (Se, 99.99\%), mercaptopionic acid (MPA, 99\%), $N, N$-dimethylacrylamde (DMA, 99\%), 4-acryloylmorpholine (AMP, 97\%), 2-hydroxyethyl acrylate (HEA, 96\%), poly(ethylene glycol) methyl ether acrylate (PEGA, Mn =480 g/mol, 99\%), and Eosin Y disodium salt (EY, 85\%) were purchased from Sigma Aldrich. N,N-diethylacrylamde (DEA, 98\%) was purchased from Tokyo Chemical Industry. All monomers were deinhibited by percolating over a basic alumina column (Acros Organics). Milli-Q water was obtained from a MilliporeSigma Milli-Q Reference Ultrapure Water Purification System. The RAFT agent 2-(n-butyltrithiocarbonate)-propionic acid (BTPA) was synthesized according to literature procedure. ${ }^{1}$

Ground State Absorption Spectroscopy. Absorbance spectra were obtained using 2-mm glass cuvettes with a Varian Cary 5000, and baseline-corrected by subtracting the absorption spectrum of neat solvent for all samples.

Photoluminescence Spectroscopy. Emission spectra were obtained using 2-mm glass cuvettes with a Horiba Fluorolog-3 spectrofluorometer in the front-face geometry, with excitation and emission slit widths of $3 \mathrm{~nm}$.

Nuclear Magnetic Resonance (NMR). NMR quantification was performed using a Bruker Avance III HD system equipped with a TXO Prodigy probe operating at $500 \mathrm{MHz}$ for ${ }^{1} \mathrm{H}$ using MeOD as a solvent. Sodium Formate was used as a reference standard.

Gel Permeation Chromatography (GPC). GPC measurements were performed on a set of Phenomenex Phenogel 5m, 1K-75K, 300 x 7.80 mm in series with a Phenomex Phenogel 5m, 10K1000K, 300 x 7.80 mm columns with HPLC grade solvents as eluents: dimethylformamide (DMF) with $0.05 \mathrm{M}$ of $\mathrm{LiBr}$ at $60{ }^{\circ} \mathrm{C}$. Detection consisted of a Wyatt Optilab T-rEX refractive index 
detector operating at $658 \mathrm{~nm}$ and a Wyatt DAWN® HELEOS ${ }^{\circledR}$ II light scattering detector operating at $659 \mathrm{~nm}$. Absolute molecular weights and polydispersities were calculated using the Wyatt ASTRA software with dn/dc values of 0.0831 for pDMA, 0.073 for pDEA, 0.0831 for pAMP, 0.0778 for pHEA, and 0.0515 for pPEGA. The dn/dc values of copolymers were determined based on mass fractions of the block components. ${ }^{2-6}$

Inductively Coupled Plasma (ICP) Mass Spectroscopy. Quantification of cadmium was accomplished using ICP-MS of acid digested samples. Specifically, samples were digested in concentrated trace nitric acid (> 69\%, Thermo Fisher Scientific, Waltham, MA, USA) and placed at $65^{\circ} \mathrm{C}$ for at least 4 hours to allow for complete sample digestion. Ultrapure $\mathrm{H}_{2} \mathrm{O}(18.2 \mathrm{M} \Omega \cdot \mathrm{cm})$ was then added to produce a final solution of 3.0\% nitric acid (v/v) in a total sample volume of 10 mL. Quantitative standards were made using a $100 \mu \mathrm{g} / \mathrm{mL}$ mixed elemental standard containing Cd (Inorganic Ventures, Christiansburg, VA, USA) which was used to create a $100 \mathrm{ng} / \mathrm{g}$ mixed element standard in 3.0\% nitric acid (v/v) in a total sample volume of $50 \mathrm{~mL}$.

ICP-MS was performed on a computer-controlled (QTEGRA software) Thermo iCapQ ICPMS (Thermo Fisher Scientific, Waltham, MA, USA) operating in STD mode and equipped with an ESI SC-2DX PrepFAST autosampler (Omaha, NE, USA). Internal standard was added inline using the prepFAST system and consisted of $1 \mathrm{ng} / \mathrm{mL}$ of a mixed element solution containing $\mathrm{Bi}$, In, 6Li, Sc, Tb, Y (IV-ICPMS-71D from Inorganic Ventures). Online dilution was also carried out by the prepFAST system and used to generate calibration curves consisting of 100, 50, 10, 1, 0.5 ng/g Cd. Each sample was acquired using 1 survey run (10 sweeps) and 3 main (peak jumping) runs (40 sweeps). The isotopes selected for analysis were 110,111,112Cd, and 115In, 209Bi (chosen as internal standards for data interpolation and machine stability). Instrument performance 
is optimized daily through autotuning followed by verification via a performance report (passing manufacturer specifications).

Synthesis and Purification of Oleate-Capped CdSe QDs. We synthesized oleate-coated CdSe QDs with diameter, $\mathrm{D}=2.8 \mathrm{~nm}$ by adding OA (3.02 mL, $9.51 \mathrm{mmol})$, ODE (10.0 mL, 31.2 mmol), and CdO (0.514 g, $4.00 \mathrm{mmol})$ to a dry, three-neck round-bottom flask and heating the reaction mixture to $270{ }^{\circ} \mathrm{C}$ under an $\mathrm{N}_{2}$ atmosphere with vigorous stirring. While the solution was heating, the selenium precursor was prepared by sonicating Se $(0.680 \mathrm{~g}, 8.6 \mathrm{mmol})$ in ODE (10 $\mathrm{mL}, 31.2 \mathrm{mmol}$ ) until a uniform suspension was formed. Once the cadmium precursor became clear, we then set the temperature to $260^{\circ} \mathrm{C}$ and rapidly injected $1 \mathrm{~mL}$ of the selenium precursor. We allowed the QDs to grow for 60 seconds before thermal quenching with a water bath. In order to extract the unused precursor, we added ethanol in a 6:1 (v/v) ratio to QDs and centrifuged the mixture at $7500 \mathrm{rpm}$ for $10 \mathrm{~min}$. We discarded the supernatant and redispersed the QDs in minimal hexanes. We repeated this washing once more and stored the final QD solution in hexanes. Please see the previous report for transmission electron micrographs (TEMs) and associated size distributions of the oleate-capped CdSe QDs prepared with this procedure. ${ }^{7}$ We determined the concentration of the QD solution by comparing the position/intensity of the first-absorbance maximum and full width at half maximum of the photoluminescence to previous reports with CdSe. $^{8}$

Phase Transfer of QDs into Water. In order to solubilize the CdSe QDs in water, we performed a ligand exchange with mercaptopropionic acid (MPA). We dried oleate-capped QDs in hexanes and resuspended them in chloroform to produce $4 \mathrm{~mL}$ of a $10 \mu \mathrm{M}$ solution in a centrifuge tube. We then added water $(1.5 \mathrm{~mL}, 83.3 \mathrm{mmol})$ on top of the chloroform layer. Directly to this water layer, we added MPA $(0.8 \mu \mathrm{L}, 9.18 \mu \mathrm{mol})$ and vigorously shook the tube for 1 minute 
to allow the QDs to phase transfer. We then added $30 \mu \mathrm{L}$ of $1 \mathrm{M}$ potassium hydroxide and shook for an additional 30 seconds. We centrifuged the tube at $7500 \mathrm{rpm}$ for 5 minutes and passed the aqueous layer through a polyvinylidene fluoride (PVDF) syringe filter with $0.2 \mu \mathrm{m}$ pores to remove aggregates.

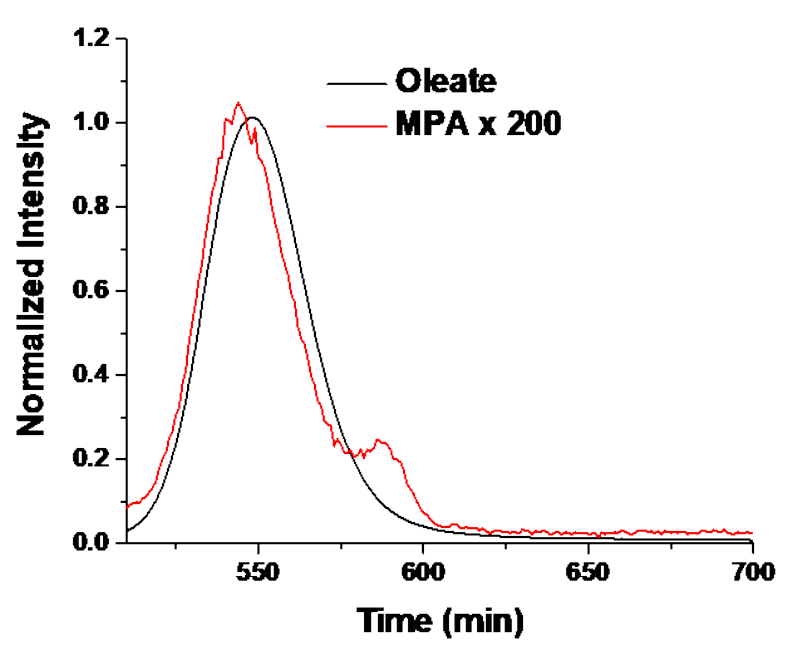

Figure S1. Fluorescence spectra for both oleate- (black) and MPA- (red) coated CdSe QDs. Spectra were normalized to the absorbance at the excitation wavelength $(390 \mathrm{~nm})$ and the MPA-CdSe spectrum was scalled by a factor of 200. We did not measure the PL QY of these QDs, but we report a decrease in PL QY of approximately two orders of magnitude when the native oleate ligands are exchanged with MPA

PET-RAFT Polymerization and Purification. To a $2 \mathrm{~mL}$ vial, we added $4.61 \mathrm{mmol}$ of monomer, BTPA (5.7 mg, $23.9 \mu \mathrm{mol})$, QDs (2 nmol) [or EY (24.1 $\mu \mathrm{g}, 34.88 \mathrm{nmol})$ ], a magnetic stir bar, and sodium formate $(0.68 \mathrm{mg}, 10 \mu \mathrm{mol})$ as an internal standard. Finally, water was added to reach a total volume of $1 \mathrm{~mL}$. We then degassed the solution by sparging with ultrapure argon gas for 5 minutes. Following degassing the sample was illuminated using a green laser (40 $\mathrm{mW} / \mathrm{cm}^{2}$ [5 mW and a $2 \mathrm{~mm}$ diameter spot size)], $532 \mathrm{~nm}$ ) while stirring to initate polymerization. In the case where CdSe QDs were the photocatalyst, we purified the resulting polymer by centrifugation using Amicon Ultra Centrifugal Filters (30 KDa pores, 3500rpm, 18 minutes) to selectively remove the QDs from the polymer and unreacted monomer. After centrifugation, we collected the filtrate and resuspended the concentrate with $33 \mu \mathrm{L}$ of a basic MPA solution 
(consisting of $3 \mathrm{mM} \mathrm{MPA}$ and $7.5 \mathrm{mM} \mathrm{KOH})$ and water $(14 \mathrm{~mL}, 778 \mathrm{mmol}$ ). After three centrifugation cycles, we combined and dried the filtrates via lyophilization. When recycling the QD photocatalyst, we would then dilute the concentrate to $0.5 \mathrm{~mL}$ with water and added $4.61 \mathrm{mmol}$ of monomer, BTPA $(5.7 \mathrm{mg}, 23.9 \mu \mathrm{mol})$, and sodium formate $(0.68 \mathrm{mg}, 10 \mu \mathrm{mol})$. The sample was then degassed, illuminated, and as described above.

When growing block copolymers, we performed the polymerization as described above and then purified out the remaining monomer by centrifugation using small-pored protein concentrators (3KDa, 3500rpm, 45 minutes). After centrifugation, we discarded the filtrate and resuspended the concentrate with $33 \mu \mathrm{L}$ of a basic MPA solution and water (10 mL, $556 \mathrm{mmol})$. After three successive purifications we collected the $1.5 \mathrm{~mL}$ of QDs/polymer and added sodium formate $(0.68 \mathrm{mg}, 10 \mu \mathrm{mol})$ and $4.61 \mathrm{mmol}$ of monomer. We then degassed and illuminated the

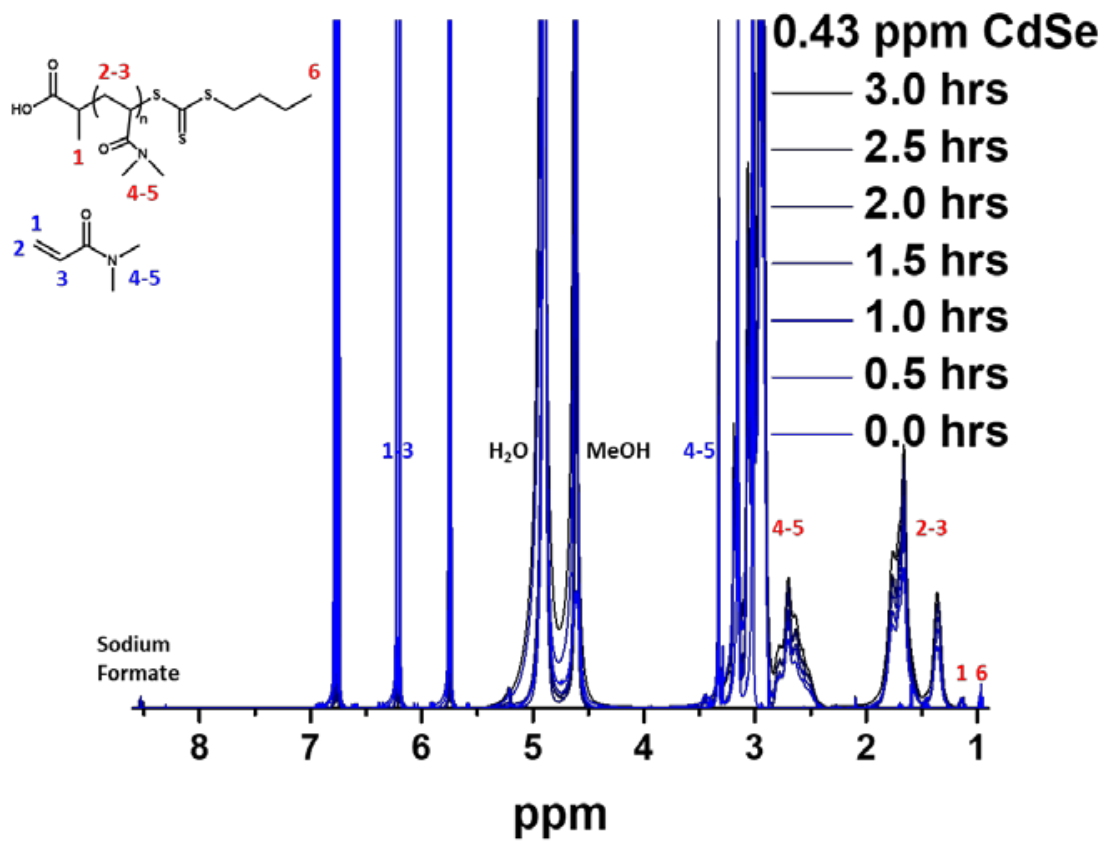

Figure S2. ${ }^{1} \mathrm{H}$ NMR spectra for the aliquots taken during the aqueous polymerization of DMA (blue to black) with $0.43 \mathrm{ppm}$ CdSe QDs shown in Figure 2A,C. Aliquots were taken from $3 \mathrm{~mL}$ of $2 \mu \mathrm{M}$ QDs, $23.9 \mathrm{mM}$ BTPA, and 4.61 M DMA and illuminated with a green laser (532 nm, $5 \mathrm{~mW}$ ) in the absence of oxygen. 
resulting solution as described previously. Finally, we followed the procedure with large pore filters to remove the QDs before analysis with GPC.

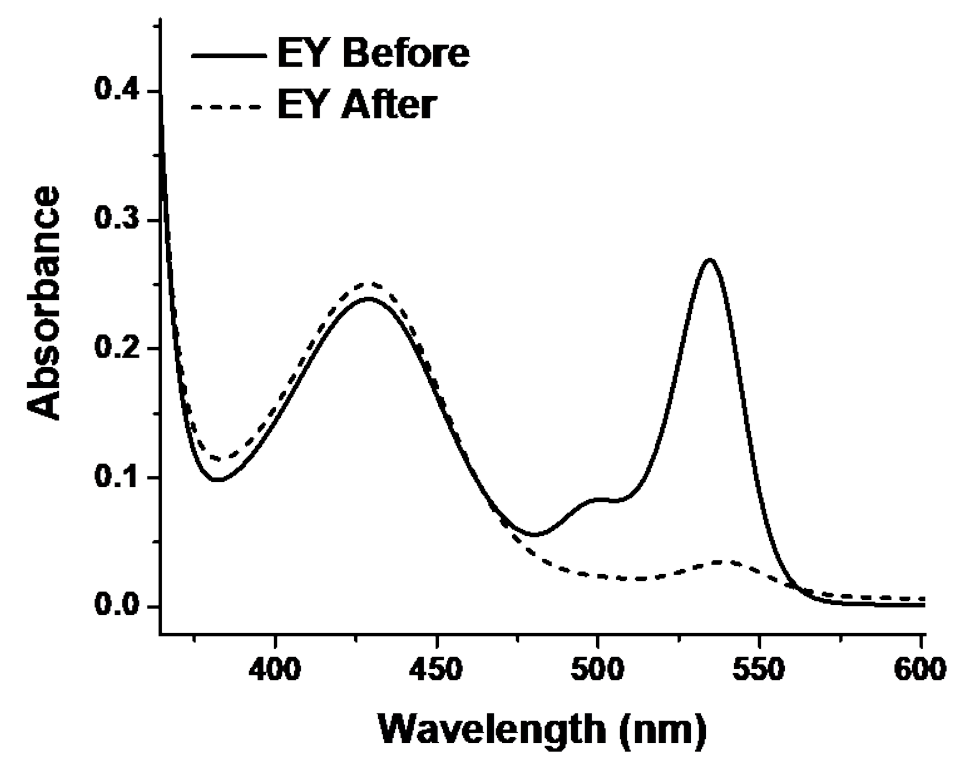

Figure S3. Absorbance spectra for 3 ppm EY before (solid) and after (dashed) 22 hours of illumination using a $5 \mathrm{~mW}, 532 \mathrm{~nm}$ green laser with $23.9 \mathrm{mM}$ BTPA and 4.61 M DMA in water.

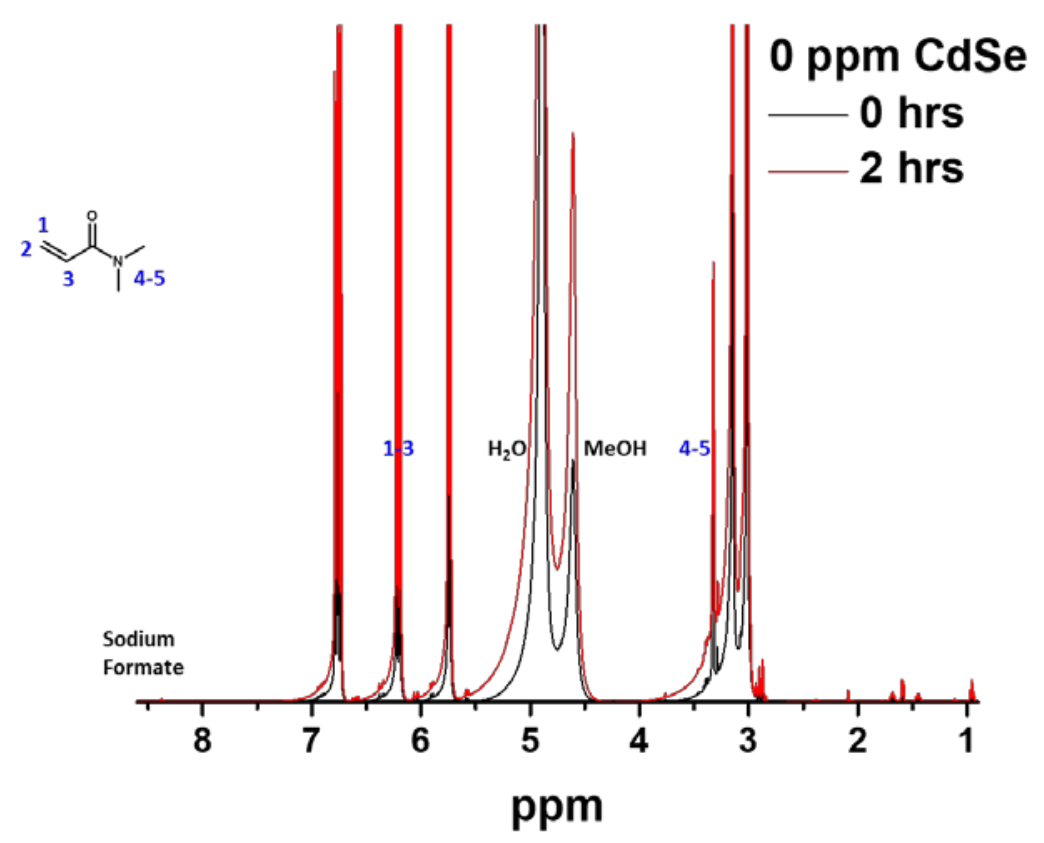

Figure S4. ${ }^{1} \mathrm{H}$ NMR spectra for the aliquots taken before (black) and after (red) the illumination of DMA with $0 \mathrm{ppm}$ CdSe QDs. Aliquots were taken from $1 \mathrm{~mL}$ of $23.9 \mathrm{mM}$ BTPA and 4.61 M DMA and illuminated with a green laser (532 nm, $5 \mathrm{~mW}$ ) in the absence of oxygen for 2 hours. 


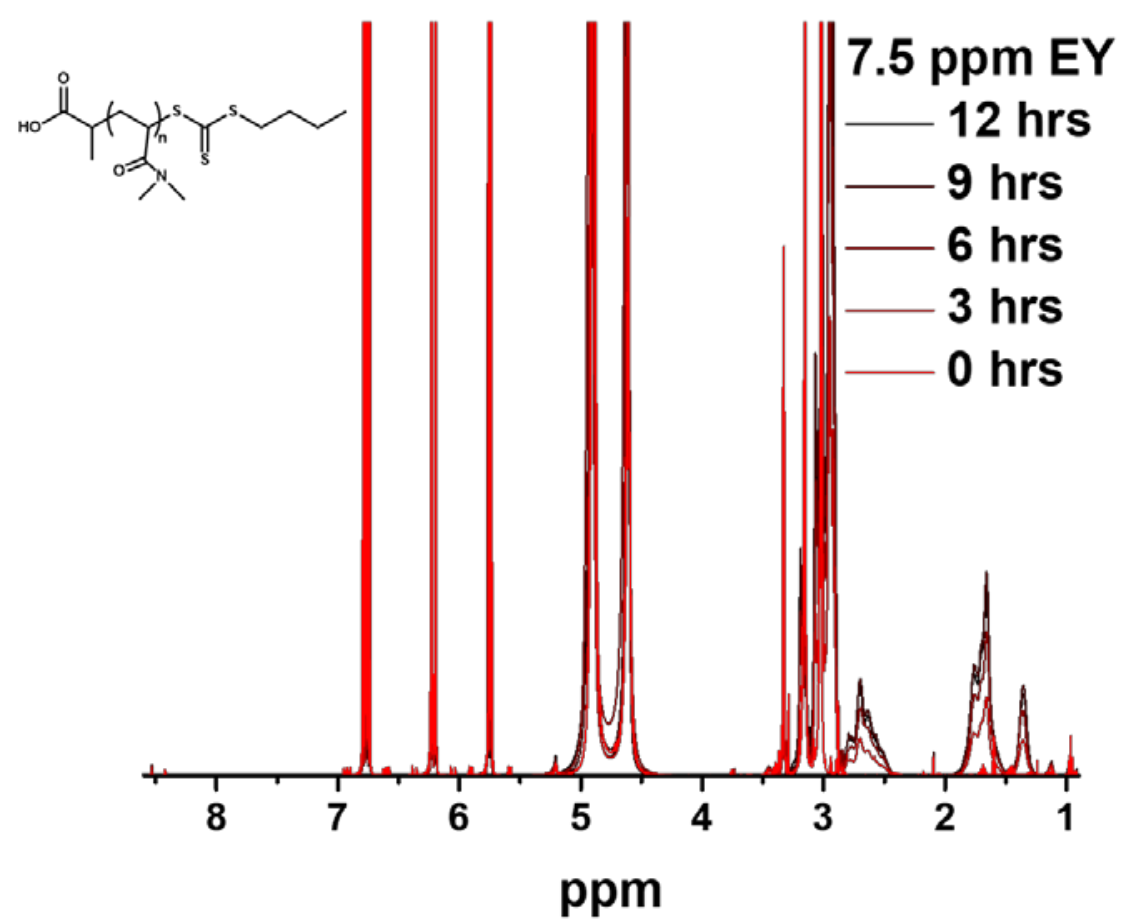

Figure S5. ${ }^{1} \mathrm{H}$ NMR spectra for the aliquots taken during the aqueous polymerization of DMA (red to black) with 7.5 ppm shown in Figure 2A. Aliquots were taken from $3 \mathrm{~mL}$ of $35 \mu \mathrm{M}$ EY, $23.9 \mathrm{mM}$ BTPA, and $4.61 \mathrm{M}$ DMA and illuminated with a green laser $(532 \mathrm{~nm}, 5 \mathrm{~mW})$ in the absence of oxygen.

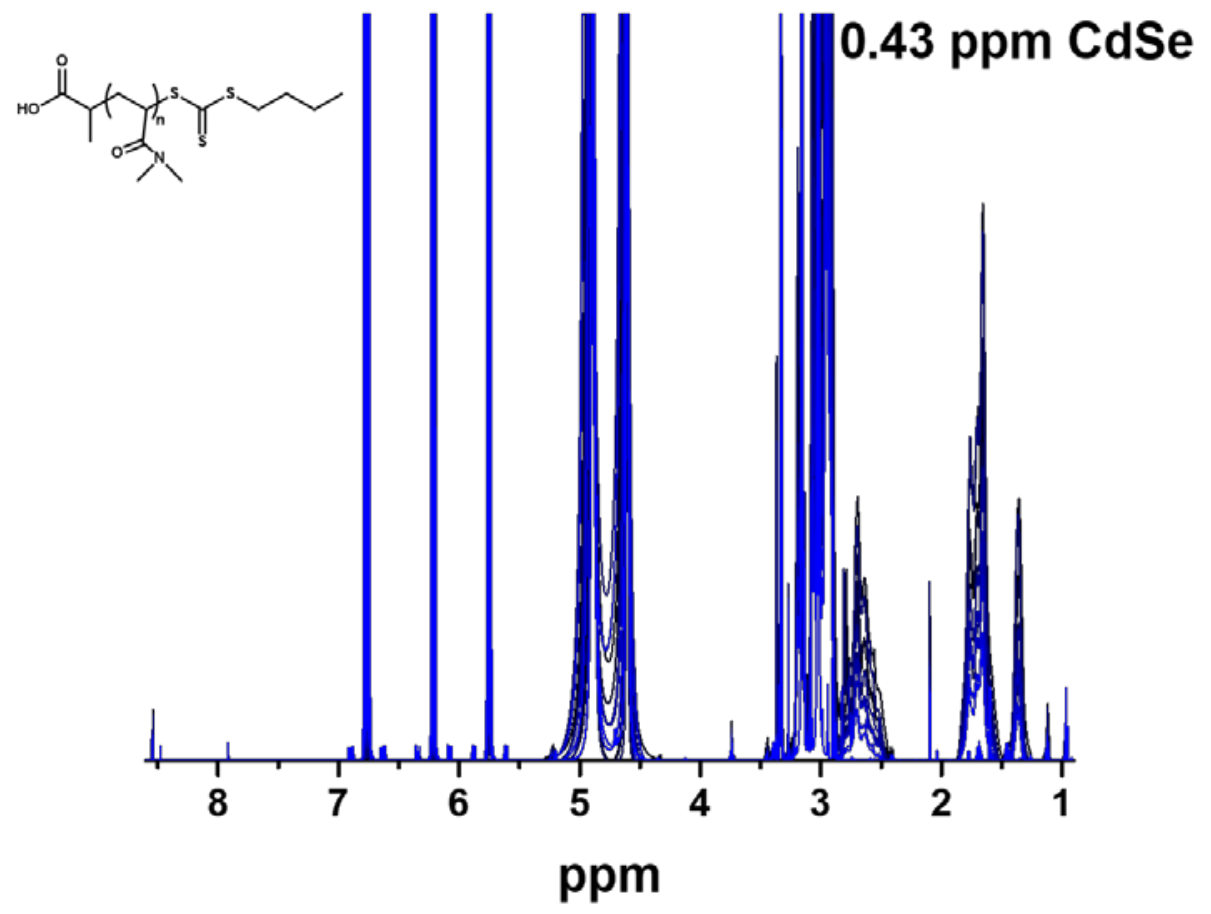

Figure S6. ${ }^{1} \mathrm{H}$ NMR spectra for the aliquots taken during the aqueous polymerization of DMA (blue to black) with $0.43 \mathrm{ppm}$ CdSe QDs with interrupted illumination shown in Figure 2B. Aliquots were taken from $3 \mathrm{~mL}$ of $2 \mu \mathrm{M}$ QDs, $23.9 \mathrm{mM}$ BTPA, and 4.61 M DMA and illuminated with a green laser (532 nm, $5 \mathrm{~mW}$ ) in the absence of oxygen. 


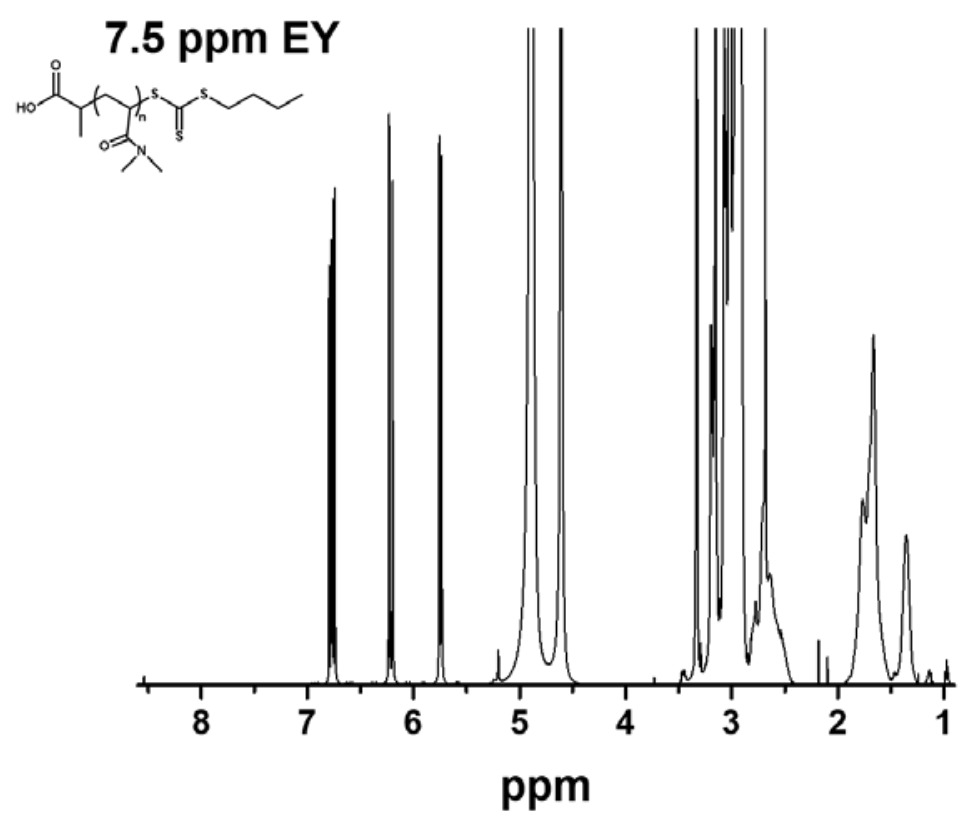

Figure S7. ${ }^{1} \mathrm{H}$ NMR spectra for the aqueous polymerization of DMA with $7.5 \mathrm{ppm}$ EY shown in Table 1. The sample was prepared with $1 \mathrm{~mL}$ of $35 \mu \mathrm{M} \mathrm{EY,} 23.9 \mathrm{mM}$ BTPA, and 4.61 M DMA and illuminated with a green laser (532 nm, $5 \mathrm{~mW}$ ) in the absence of oxygen for 22 hours.

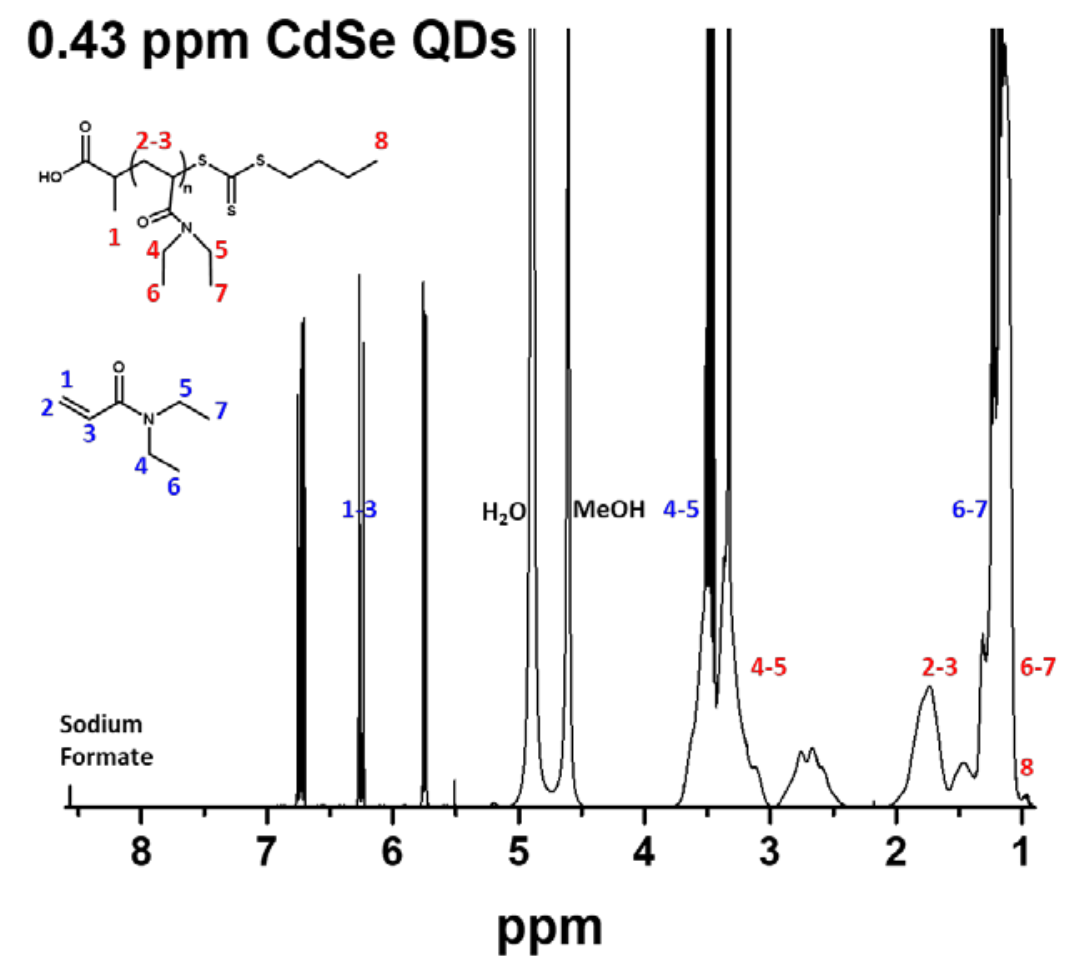

Figure S8. ${ }^{1} \mathrm{H}$ NMR spectra for the aqueous polymerization of DEA with $0.43 \mathrm{ppm}$ CdSe QDs shown in Table 1. The sample was prepared with $1 \mathrm{~mL}$ of $2 \mu \mathrm{M}$ QDs, $23.9 \mathrm{mM}$ BTPA, and 4.61 M DEA and illuminated with a green laser (532 nm. $5 \mathrm{~mW}$ ) in the absence of oxvgen for 2 hours. 


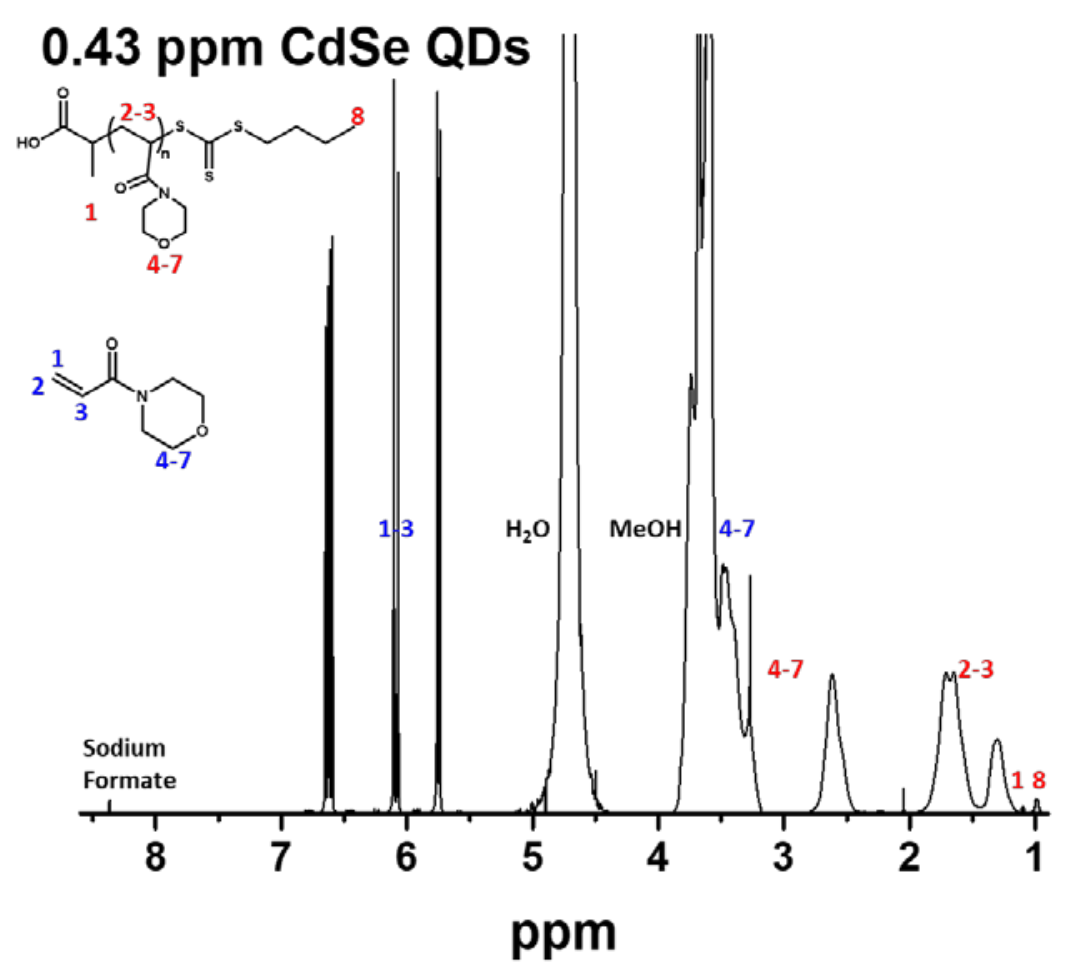

Figure S9. ${ }^{1} \mathrm{H}$ NMR spectra for the aqueous polymerization of AMP with 0.43 ppm CdSe QDs shown in Table 1. The sample was prepared with $1 \mathrm{~mL}$ of $2 \mu \mathrm{M}$ QDs, $23.9 \mathrm{mM}$ BTPA, and 4.61 M AMP and illuminated with a green laser (532 nm, $5 \mathrm{~mW}$ ) in the absence of oxygen for 2 hours.

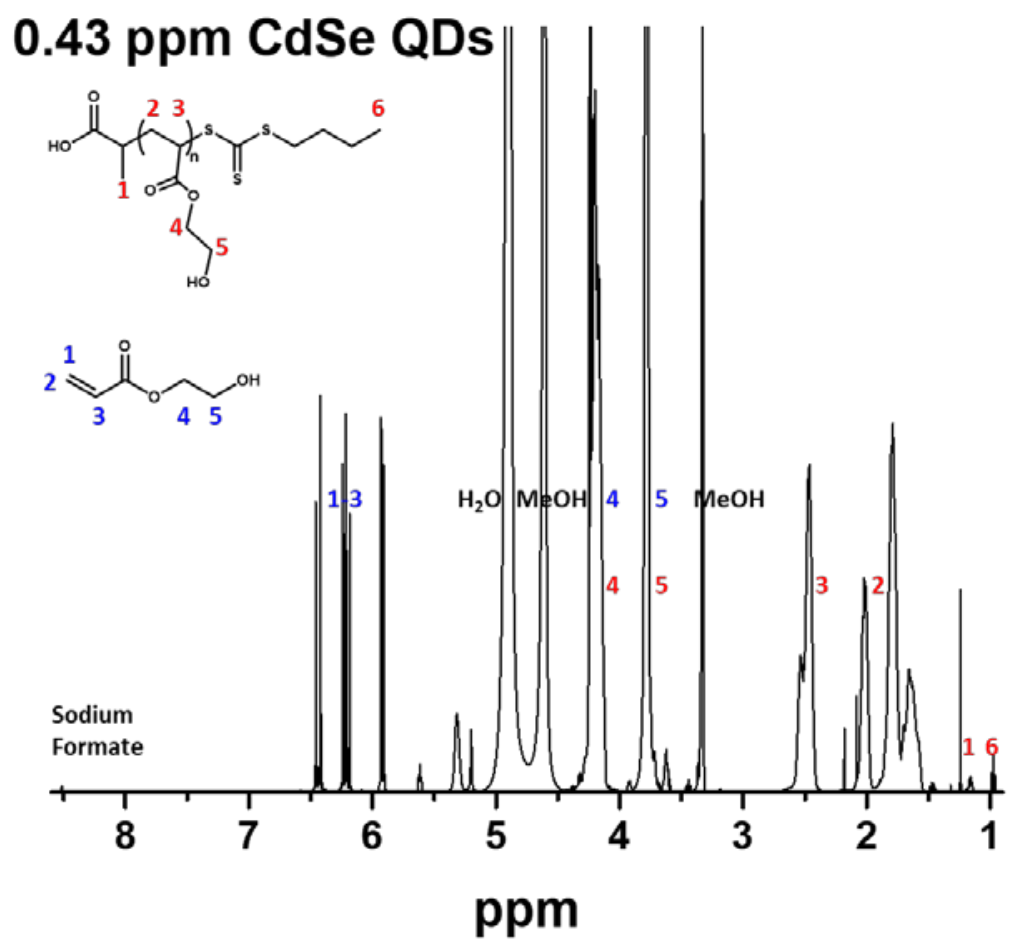

Figure S10. ${ }^{1} \mathrm{H}$ NMR spectra for the aqueous polymerization of HEA with 0.43 ppm CdSe QDs shown in Table 1. The sample was prepared with $1 \mathrm{~mL}$ of $2 \mu \mathrm{M}$ QDs, $23.9 \mathrm{mM}$ BTPA, and 4.61 M HEA and illuminated with a green laser (532 nm, $5 \mathrm{~mW}$ ) in the absence of oxygen for 2 hours. 


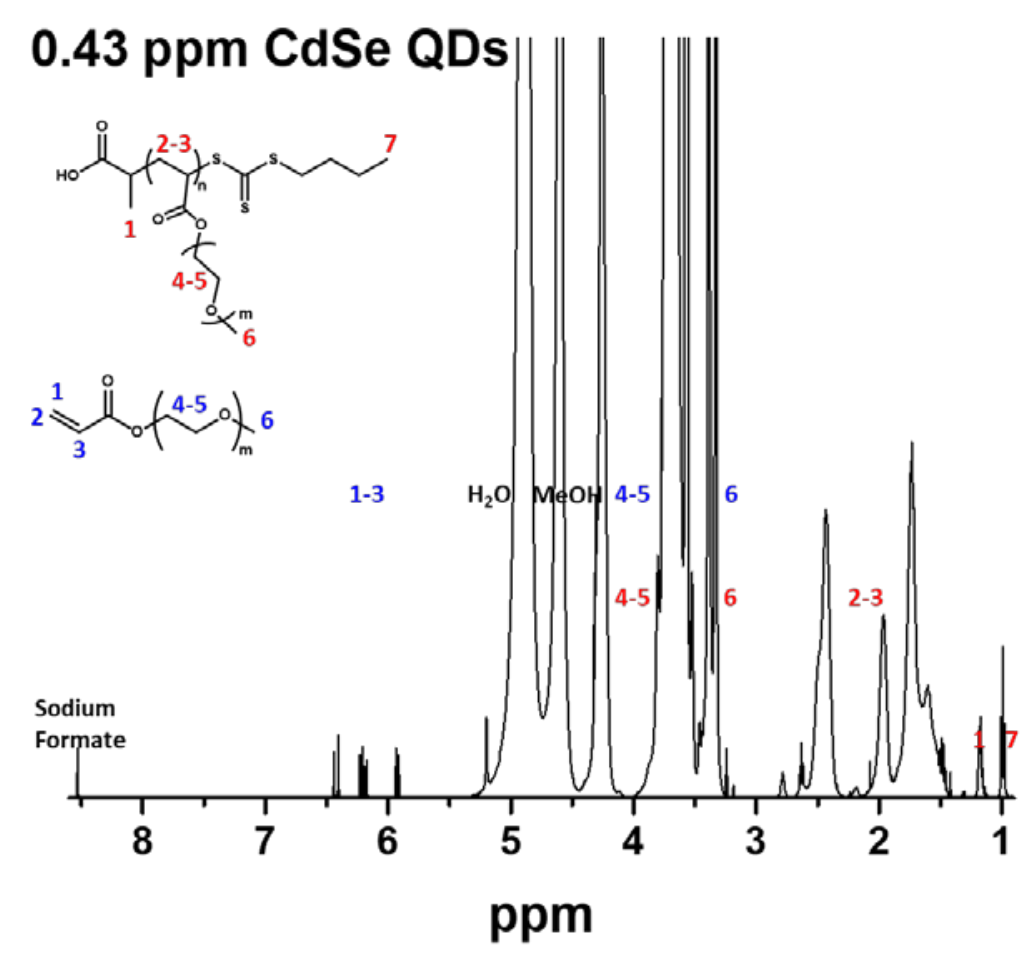

Figure S11. ${ }^{1} \mathrm{H}$ NMR spectra for the aqueous polymerization of PEGA with $0.43 \mathrm{ppm}$ CdSe QDs shown in Table 1. The sample was prepared with $1 \mathrm{~mL}$ of $2 \mu \mathrm{M}$ QDs, $23.9 \mathrm{mM}$ BTPA, and $4.61 \mathrm{M}$ DMAEA and illuminated with a green laser (532 nm, $5 \mathrm{~mW}$ ) in the absence of oxygen for 2 hours.

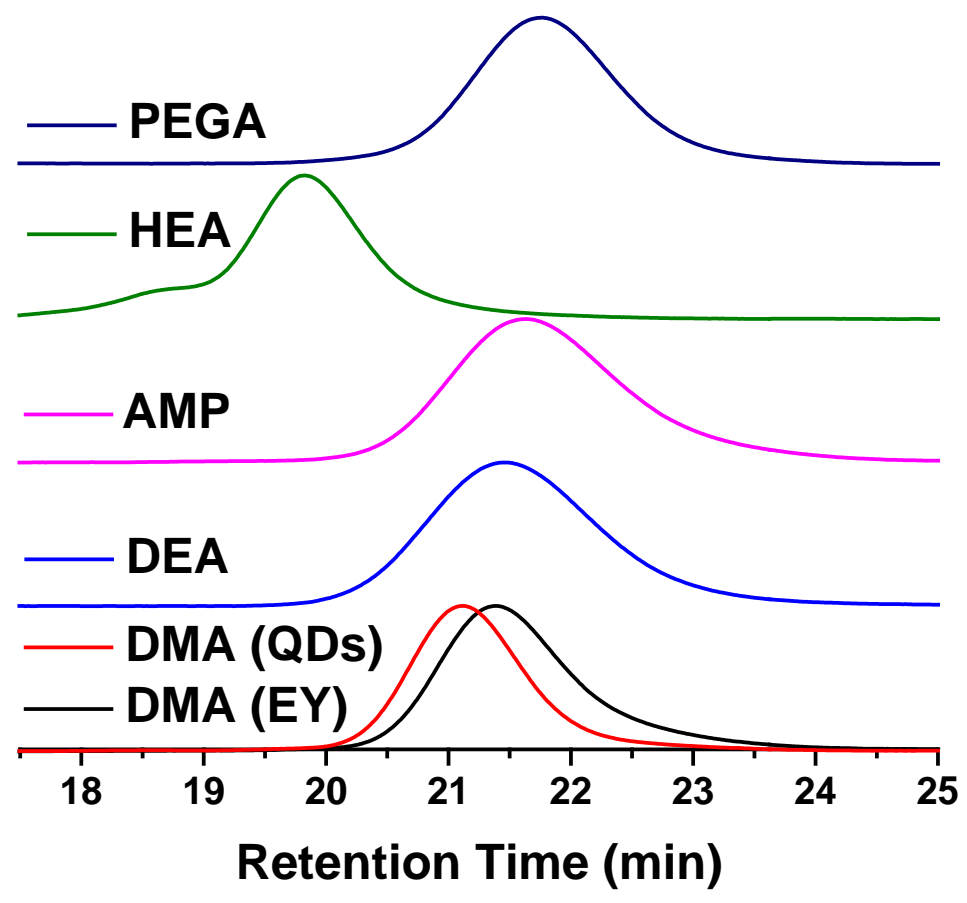

Figure S12. GPC chromatograms for the aqueous polymerization with $0.43 \mathrm{ppm}$ CdSe QDs or $7.5 \mathrm{ppm}$ EY for all monomers shown in Table 1. The sample was prepared with $1 \mathrm{~mL}$ of $2 \mu \mathrm{M}$ QDs (or $35 \mu \mathrm{M} \mathrm{EY}$ ), $23.9 \mathrm{mM}$ BTPA, and 4.61 M monomer (or 1 M PEGA) and illuminated with a green laser (532 nm, $5 \mathrm{~mW}$ ) in the absence of oxygen for 2 (or 22) hours. 


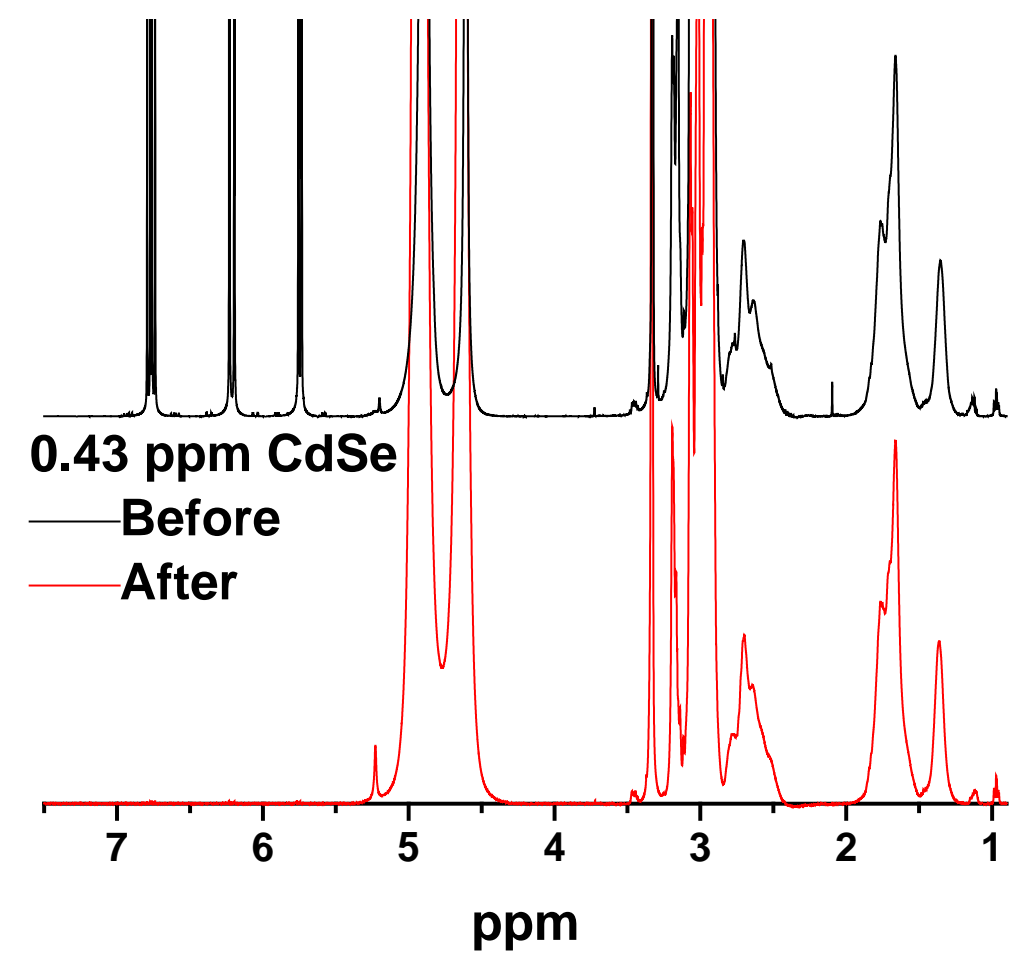

Figure S13. ${ }^{1} \mathrm{H}$ NMR spectra for pDMA before (black) and after (red) 3 rounds of purification with 3KDa Amicon Ultra protein concentrators to remove the monomer from the QD/Polymer mixture.

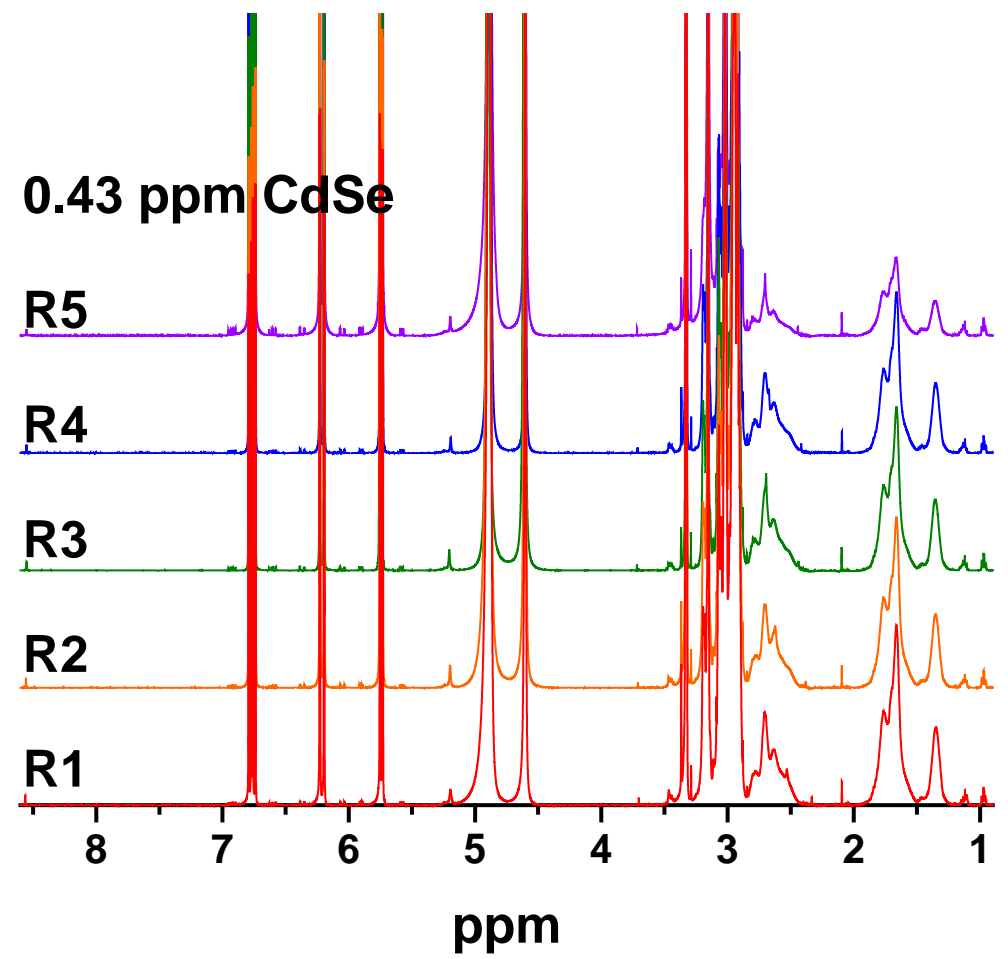

Figure S14. ${ }^{1} \mathrm{H}$ NMR spectra for the aqueous polymerization of DMA with $0.43 \mathrm{ppm}$ recycled CdSe QDs shown in Table 2. The sample was prepared with $1 \mathrm{~mL}$ of $3 \mu \mathrm{M}$ QDs, $23.9 \mathrm{mM}$ BTPA, and 4.61 M DMAEA and illuminated with a green laser ( $532 \mathrm{~nm}, 5 \mathrm{~mW}$ ) in the absence of oxygen for 1.5 hours. 


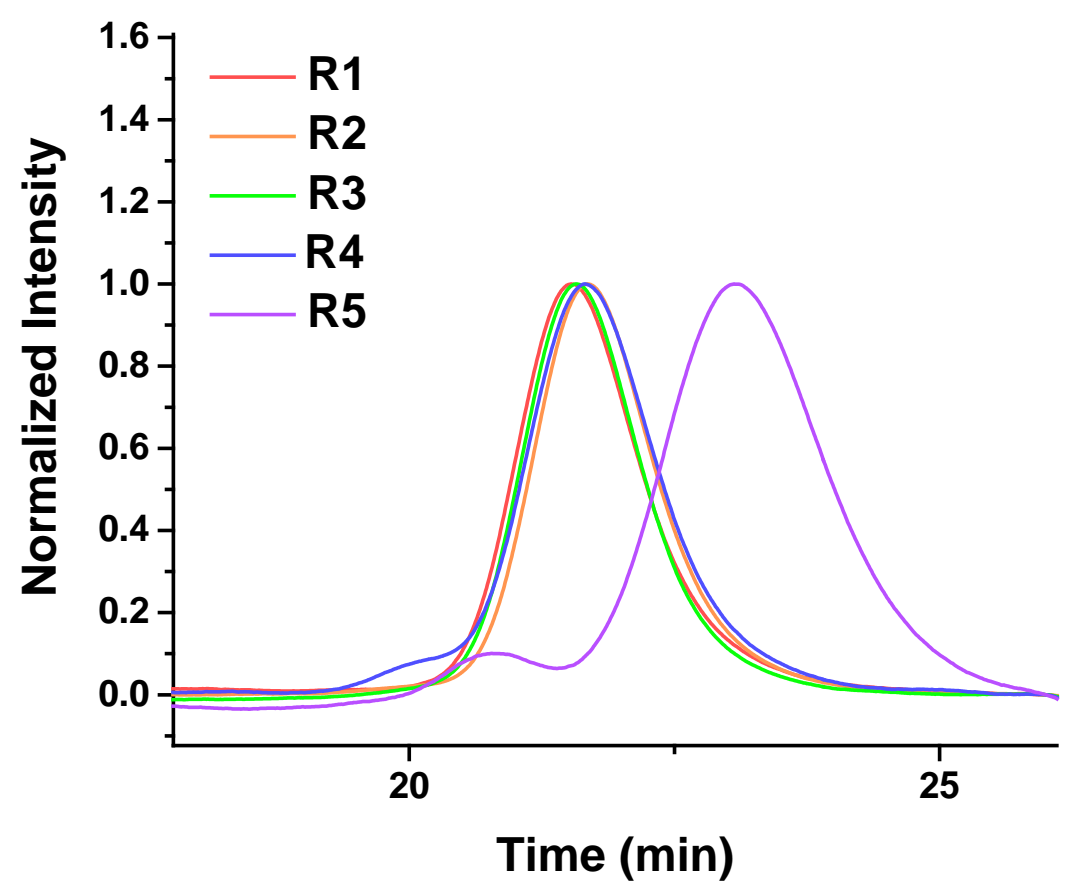

Figure S15. GPC chromatograms for the aqueous polymerization of DMA with 0.43 ppm recycled CdSe QDs shown in Table 2. The sample was prepared with $1 \mathrm{~mL}$ of $3 \mu \mathrm{M}$ QDs, $23.9 \mathrm{mM}$ BTPA, and 4.61 M DMAEA and illuminated with a green laser $(532 \mathrm{~nm}, 5 \mathrm{~mW})$ in the absence of oxygen for 1.5 hours.

\section{References}

1. Niu, J.; Lunn, D. J.; Pusuluri, A.; Yoo, J. I.; O'Malley, M. A.; Mitragotri, S.; Soh, H. T.; Hawker, C. J., Engineering live cell surfaces with functional polymers via cytocompatible controlled radical polymerization. Nature Chem. 2017, 9, 537.

2. Ridge, O.; Industries, E.; Livermore, L.; Office, P., (12) Patent Application Publication (10) Pub. No.: US 2017/0096730 A1. 2017, 1 (19).

3. $\quad$ Mendonça, P. V.; Lima, M. S.; Guliashvili, T.; Serra, A. C.; Coelho, J. F. J., Deep eutectic solvents (DES): Excellent green solvents for rapid SARA ATRP of biorelevant hydrophilic monomers at ambient temperature. Polymer 2017, 132, 114-121.

4. Grazon, C.; Rieger, J.; Sanson, N.; Charleux, B., Study of poly(N,N-diethylacrylamide) nanogel formation by aqueous dispersion polymerization of N,N-diethylacrylamide in the presence of poly(ethylene oxide)-b-poly(N,N-dimethylacrylamide) amphiphilic macromolecular RAFT agents. Soft Matter 2011, 7 (7), 3482-3490.

5. Lamb, J. R.; Qin, K. P.; Johnson, J. A., Visible-light-mediated, additive-free, and opento-air controlled radical polymerization of acrylates and acrylamides. Polym. Chem. 2019, 10 (13), 1585-1590.

6. $\quad$ Chen, M.; Inglefield, D. L.; Zhang, K.; Hudson, A. G.; Talley, S. J.; Moore, R. B.; Long, T. E., Synthesis of urea-containing ABA triblock copolymers: Influence of pendant hydrogen bonding on morphology and thermomechanical properties. J. Polym. Sci. Pol. Chem. 2018, 56 (16), 1844-1852. 
7. Flamee, S.; Cirillo, M.; Abe, S.; De Nolf, K.; Gomes, R.; Aubert, T.; Hens, Z., Fast, High Yield, and High Solid Loading Synthesis of Metal Selenide Nanocrystals. Chem. Mater. 2013, 25 (12), 2476-2483.

8. $\quad \mathrm{Yu}, \mathrm{W}$. W.; Qu, L.; Guo, W.; Peng, X., Experimental Determination of the Extinction Coefficient of CdTe, CdSe and CdS Nanocrystals. Chem. Mater. 2004, 16 (3), 560-560. 